\title{
Performanz als Ausweg? Der Videoclip 99 Problems von Jay-Z und Mark Romanek
}

\author{
What can be shown, what should not be shown - \\ few issues arouse more public clamor. ${ }^{1}$
}

Es kommt nicht allzu oft vor, dass sich Interpretinnen oder Interpreten im Rahmen eines Musikvideos präsentieren, an dessen Ende die Darstellung ihrer eigenen Ermordung steht. Nicht, dass die Inszenierung des Todes der im Clip gespielten Figur ein absolutes Unikum darstellen würde: In dem von Rocky Schenck 1995 zu Nick Caves Where The Wild Roses Grow gedrehten Video verkörperte die als Gaststar auftretende Sängerin Kylie Minogue zum Beispiel den Part einer jungen Frau, die einem von Cave dargestellten Lustmörder zum Opfer fällt. ${ }^{2}$

1 Sontag, Susan, Regarding the Pain of Others, New York, 2003, S. 69.

2 Dies ist nur ein Beispiel, an das sich eine ganze Phalanx von weiteren, zuvor wie später entstandenen Videos anfügen ließe. Nur um den Radius möglicher Fälle anzudeuten, seien hier eher wahllos sechs weitere Clips angeführt: In dem von John Hillcoat gedrehten Video zu dem Song You're Not Alone der britischen Gruppe Embrace (März 2000) stürzt die Band von einem über einen Wasserfall gespannten Hochseil; ebenfalls von John Hillcoat stammt die (ausgerechnet für den amerikanischen Markt gedrehte) Version zu dem Embrace-Song All You Good Good People (August 1998), in dem der Sänger der Gruppe einen Verurteilten spielt, der auf dem elektrischen Stuhl endet (allerdings wird sein Tod hier nicht direkt gezeigt, sondern - wie bereits in Andy Morahans Videoclip zu Don't Cry von den Guns N'Roses aus dem Jahr 1991 - mit einer Geburt parallelisiert); das von Sam Brown zu dem Titel Fallen Angel der Band Elbow gedrehte Video (Juli 2003) zeigt die Mordphantasien, die deren Sänger seinen Kollegen gegenüber hegt - am Schluss des Clips wird er dann selbst von einem herabstürzenden Klavier erschlagen; das deutsche Regisseurteam Alex \& Liane deutet an, dass die Mitglieder der Gruppe Madsen im Rahmen ihres Clips zu Immer mehr (Herbst 2005) sich verlebendigenden Dinosaurier-Exponaten zum Opfer fallen. Ähnlich wie die Sängerin in Rocky Schencks oben erwähntem Video zu dem Song von Nick Cave und Kylie Minogue sind die Mitglieder der von der Formation Blanche gespielten Band in dem Video zu dem Stück Someday bereits tot, wenn der im November 2004 entstandene Clip beginnt; der in Schwarzweiß-Szenen gezeigte Auftritt der Musiker in einer Kirche wird somit als eine Art Geisterstunde ausgewiesen; diesem werden die farbig gehaltenen Aufnahmen gegenüber gestellt, in denen die diversen Unfälle vorgeführt werden, durch welche die Musiker zu Tode kamen. In dem im Februar 2006 von Chris Milk vorgelegten Video zu Touch The Sky von Kanye West spielt der Interpret den nach dem Vorbild von Evil Knievel gestalteten Stuntman Evel Kanyevel, der am Schluss des Clips mit seiner Rakete abstürzt. Da sich die Trickaufnahmen des Absturzes jedoch bewusst als solche ausweisen und West im letzten Bild des Clips lebend zu sehen ist, wird der ,Tod' des Stuntman in gewisser Weise vom realen Leben des Interpreten abgekoppelt, der Kanyevel in dem Video sozusagen stellvertretend diverse problematische Episoden der eigenen Biografie aufbürdet (West überlebte 2002 nur knapp einen Autounfall; in dem Clip werden Anspielungen auf seine negativen Kommentare zu Präsident Bush nach dem Wüten des Hurrikan „Katrina“ gemacht; außerdem finden sich Wests Ängste vor durch Erfolg verursachter Selbstentfremdung und 
Üblicherweise werden derartige Darstellungen sodann jedoch durch nachfolgende Videos konterkariert, die klar stellen, dass zwischen der eingenommenen Rolle und der dahinter stehenden, realen Person ein Unterschied besteht: Kommt die verkörperte Figur im einen Clip zu Tode, so spielt deren Darstellerin oder Darsteller im nächsten schon wieder einen ganz anderen, neuen Charakter.

So fand auch Kylie Minogues Interpretation einer Ermordeten mitten in ihrer Karriere statt, und eben darin unterscheidet sich diese Darstellung von derjenigen, die Mark Romanek im April 2004 vorlegte: Im Rahmen des Videos, das er zu dem Stück 99 Problems des Hip-Hop-Musikers Jay-Z drehte, inszenierte Romanek am Ende des Clips die Erschießung des Rappers. Indem dieser Clip den zuvor beschlossenen Rückzug des Interpreten aus dem Musikgeschäft markierte, konnte sein dort gezeigter, Tod' zunächst durch kein nachfolgendes Video des Künstlers relativiert werden. ${ }^{3}$ Zudem spielte Minogue in Where The Wild Roses Grow eindeutig eine von ihrer Person unabhängige Rolle, während Jay-Z in 99 Problems als er selbst auftritt: eine Identitätskonstruktion, die zudem noch durch den Umstand betont wurde, dass der gesamte, in Schwarzweiß gedrehte Clip mit seiner instabilen Kameraführung und seinen scheinbar kunstlosen Bildausschnitten zum Teil der Ästhetik von Dokumentaraufnahmen folgt ${ }^{4}$ (eine wiederholt in das Video eingeschnittene Aufnahme einer vor das Kameraobjektiv gehaltenen Hand suggeriert zum Beispiel, dass hier einer der Bewohner des New Yorker Stadtteils Brooklyn die Dreharbeiten behindern möchte).

In seiner Koinzidenz von tatsächlichem Karriere-Ende und vermeintlichem Tod ist Romaneks Clip am ehesten noch dem letzten Video vergleichbar, das Nicholas Brandt im März 1996 für die Boygroup Take That drehte, bevor deren Mitglieder getrennte Wege gingen: Gezeigt wird, wie eine junge Frau (ein fanatischer Fan, wie zunächst anzunehmen ist), welche die vier Sänger entführt zu haben scheint, die jungen Männer zu der von ihnen interpretierten, sanften Ballade How Deep Is Your Love auf Stühle fesselt und den Leadsinger schließlich in einen Abgrund stößt.

einem plötzlichen Karriereende dort ebenfalls thematisiert). Vgl. dazu http://musicvideowire.com/ dynamic/article_view.asp?AID=11639 [Stand: 12. 3. 2007]. Die Angaben zu Regisseur und Entstehungsdatum folgen im Weiteren - sofern nicht ausdrücklich vermerkt - der von Alex Garcia seit 1998 gepflegten Online-Datenbank „Music Video DataBase“ unter http://www.mvdbase.com [Stand: 12. 3. 2007]. Ich möchte an dieser Stelle Kevin Holy (Athens, Ohio) und Miguel Martinez (Madrid) danken, die mir bei der Suche nach dieser Art von Videoclips behilflich waren.

3 Nach der Veröffentlichung des Videos zu 99 Problems wirkte Jay-Z als Gaststar u. a. bei dem im November 2004 veröffentlichten Clip der Gruppe Linkin Park mit, der die Live-Aufführung der Stücke Numb und Encore dokumentierte; Regie führten hier Joe DeMaio und Kimo Proudfoot. Inzwischen hat sich der 2004 verkündete Abschied Jay-Zs jedoch als tatsächliche Pause erwiesen: Am 21. 11. 2006 erschien sein neues Album Kingdom Come; mehrfach thematisiert Jay-Z dort seine Rückkehr, so u. a. in der Zeile des Titeltracks: „Gotta admit a lil bit / I was sick of rap / But despite that / The boy is back".

4 Daher fühlte sich Jay- $Z$ auch dazu herausgefordert, in dem unten zitierten Interview Sway Calloways mit Jay-Z (wie Anm. 10), die Tatsache zu betonen, dass er in dem Clip - wie ein Schauspieler - lediglich eine Rolle spielte. 
Auch hier visualisierte der fiktive Mord ein faktisches Ende innerhalb des Musikbetriebs. $^{5}$

Doch im Unterschied dazu zeigt Romaneks 99 Problems den Tod Jay-Zs nicht nur explizit, sondern betont ihn zusätzlich durch die Verwendung von Zeitlupe und Zwischenschnitten. Dies hebt die Sequenz auch deutlich von dem Video ab, das Baillie Walsh 1998 zu dem Stück Come Together der Gruppe Spiritualized drehte und das in gewisser Hinsicht als Vorläufer zu Romaneks Clip betrachtet werden kann. Denn der Leadsänger von Spiritualized spielt hier einen verhafteten Verbrecher, der zum Ende des Videos vor einem Gerichtsgebäude von einer jungen Frau in einem Akt der Selbstjustiz niedergeschossen wird. Anders als in Romaneks Video vollzieht sich dieser Anschlag jedoch innerhalb von Sekundenbruchteilen, während Jay-Z in dem.Clip zu 99 Problems unter einem anhaltenden Kugelhagel langsam zu Boden geht (insgesamt zieht sich die Erschießung - mit Zwischenschnitten - über 25 Sekunden hin). Während zudem in dem Video zu Come Together nichts auf die am Ende erfolgende Tat vorausweist (zu sehen ist lediglich, wie der Verhaftete zum Gericht eskortiert wird), deuten in 99 Problems viele der zuvor gezeigten Szenen latent drohender ${ }^{6}$ wie offen ausbrechender Gewalt ${ }^{7}$ auf den tödlichen Anschlag hin, so dass dieser den Höhepunkt eines sorgfältig aufgebauten Spannungsbogens darzustellen scheint. Ist mit dem Mord aber auch jener „unforgettable emotional impact" gemeint, den Romanek in seinem Treatment aus der Planungsphase des Clips zu dessen letztendlichem Ziel erklärt? ${ }^{2}$ Wäre das Auftreffen der Jay-Z tötenden Projektile somit das vordergründige Korrelat zu dem „impact", den das Video in die Emotionen des Betrachters haben soll?

Dass man beim Musiksender MTV tatsächlich die Sorge hegte, die im Clip gezeigte Erschießung Jay-Zs könne als spektakuläres und Gewalt verherrlichendes Lockmittel missverstanden werden, zeigt der Umstand, dass man ihn nur unter

5 Diesen Zusammenhang übersieht Tanja Busse, weshalb die dargestellte Situation sie ratlos zurücklässt: „Das Video bietet keine Hinweise, warum sich die Jungen in dieser mißlichen Lage befinden. Es gibt keine Erklärung [...]. Die Rolle der vier Teenager-Stars von Take That ist ungewohnt." Busse, Tanja, Mythos in Musikvideos, Münster, 1996, S. 87. Bei dem von der Gruppe vorgetragenen Song handelt es sich um die Coverversion eines Bee Gee-Klassikers von 1977 aus dem Soundtrack zu John Badhams Film Saturday Night Fever, in dem gegen Ende des Films ein Junge von einer Brücke abstürzt. Möglicherweise rekurriert der von Clipregisseur Brandt gezeigte Schluss auch hierauf. Auch Take That haben - wie Jay-Z (siehe Anm. 3) - im Jahr 2006 ein Comeback unternommen.

6 Ein Junge maskiert sich offenbar für einen Raubüberfall (Abb. 4 a, b); ein Zuhälter mustert beim Zählen der Einnahmen die von ihm kontrollierten Prostituierten mit strengem Blick; Dinge werden auf die Kamera zugeschleudert bzw. - siehe oben - die Dreharbeiten werden anscheinend behindert; Jay- $Z$ wird von einer Polizeistreife kontrolliert und bedroht; einige Szenen spielen in einem Gefängnis.

7 Der Zuhälter wird gegenüber den Prostituierten aggressiv und verfolgt sie durch die Straßen; bei einem Basketballspiel kommt es zwischen Jugendlichen zu einer Schlägerei; auf der Straße tragen zwei Männer einen Faustkampf aus.

8 Vgl. das Treatment auf Romaneks Website unter http://www.markromanek.com/video/22.html [Stand: 12. 3. 2007]; das geplante Video wird dort mit den beschließenden Worten charakterisiert: "the final effect should have an unforgettable emotional impact. think of it as ,reality-tv' with soul." 
ganz bestimmten Bedingungen ausstrahlte. Denn im Unterschied zu den meisten der ähnlich konzipierten Videos war Romaneks 99 Problems als ein Clip gedacht, der in den Programmen der Musiksender auf heavy rotation, das heißt tagsüber mindestens einmal pro Stunde laufen sollte, während ein Video wie das oben Erwähnte zu dem Song Come Together der Gruppe Spiritualized seltener und vor allem meistens im Spätprogramm zur Ausstrahlung kam. Eben diese Platzierung von 99 Problems in der heavy rotation konfrontierte die Verantwortlichen bei MTV mit der Frage, wie Romaneks Video gesendet werden konnte. Ihre diesbezüglichen Antworten machten es dem Regisseur zum einen zur Auflage, einige Details in ausgewählten Szenen durch optische Effekte und akustische Leerstellen unkenntlich zu machen (dies betrifft etwa obszöne Worte des Liedtextes sowie entsprechende Gesten), den Clip passagenweise umzuschneiden (in einer Einstellung war zum Beispiel ursprünglich ein von einem Fenster aus auf eine Passantin zielender Jugendlicher mit einer Pistole zu sehen $)^{9}$ sowie die Erstausstrahlung des Videos Ende April 2004 mit einer Erklärung durch gleich zwei MTV-Moderatoren zu versehen: Zunächst führte John Norris in die Problematik des Clips ein, indem er zum einen daran erinnerte, dass „precipitated by rising gun violence amongst young people, MTV has for more than a decade now discouraged videos containing any kind of gun imagery or fortuitous violence." Zum anderen aber wies er auf die Beweggründe für eventuelle diesbezügliche Ausnahmen hin: „On very rare occasions, however, when compelled by the artistic merits of a particular clip, MTV has offered at least some form of limited airplay to just a handful of controversial videos." Norris führte sodann einzelne Beispiele an und fügte dieser Liste schließlich Jay-Zs 99 Problems hinzu, womit er zu einem Interviewausschnitt überleitete, der den MTV-Korrespondenten, Produzenten und Rapper Sway Calloway im Gespräch mit Jay-Z zeigte. ${ }^{10}$ Als wäre er besorgt, dass man die dort formulierte Anweisung zum richtigen Verständnis des Clips nicht begreifen würde, fasste Norris die zentralen Aussagen des Gesprächs vorab schon einmal zusammen: „Jay insists that the dramatic conclusion to his music video career is not fortuitous but rather [...] ripe with symbolism, showing the death of Jay-Z and the rebirth of Shawn Carter" (Jay-Zs , bürgerlicher" oder ,privater ${ }^{6}$ Name). ${ }^{11}$

9 Auf der im Oktober 2005 erschienenen DVD The work of director Mark Romanek präsentiert der Regisseur einen unzensierten „Director's Cut" des Videos, in den diese und andere zuvor geschnittene Szenen wieder eingefügt sind.

10 Das komplette Interview wurde, durch den von Shaheem Reid geschriebenen Artikel „Jay-Z: 99 problems, hundreds of rumors" eingeleitet, am 27. 4. 2004 auch unter http://www.mtv.com/ bands/j/jay_z/99_problems/veröffentlicht.

11 Vgl. dazu auch den in der vorangehenden Anmerkung zitierten Artikel mit der Formulierung: „Jigga [Spitzname für Jay-Z] isn't promoting violence or making some Tupac-like prophecy. Rather, he used his staged death as symbolism." Gegen Ende des Clips sieht man jedoch einen Mann in einem Sarg liegen, der deutliche Ähnlichkeiten zu Tupac Amaru Shakur aufweist. Bei diesem handelt es sich um einen der vielen Musiker, die in den vergangenen Jahren den Rivalitäten zwischen RapGangs der Ost- und der Westküste zum Opfer fielen - der Schauspieler und Interpret wurde am 7. 9. 1996 in Las Vegas angeschossen und verstarb kurz darauf. 
Man mag es als paradox empfinden, dass vergleichsweise harmlose Szenen wie der mit der Waffe hantierende Jugendliche oder die obszönen Gesten aus dem Clip getilgt wurden, während man die Ermordung Jay-Zs unangetastet ließ (denkbar wäre auch eine starke Verkürzung der Sequenz gewesen, indem man die Erschießungsaufnahmen durch alternative Bilder ersetzt hätte). ${ }^{12}$ Doch zeigt diese Akzeptanz, dass man (wie auch explizit von Norris formuliert) die Szene aufgrund ihres symbolischen Gehalts als zentrales Element des Clips erkennen und anerkennen musste.

Erreicht wird dies vor allem mittels ihrer formalen Gestaltung: Durch das abstrahierende Schwarzweiß der Bilder, die Länge der Sequenz, ihre Durchsetzung mit anderen, zwischengeschnittenen Bildern, die Wiedergabe in Zeitlupe sowie durch Details wie das Fehlen von Blut oder die malerisch Funken um Jay-Z schlagenden Einschüsse (Abb. 1) erhält der ganze Vorgang etwas von einem Ballett ${ }^{13}$, was ihn seiner eigentlichen Brutalität entkleidet. Anders als bei dem SpiritualizedVideo zu Come Together, das den Betrachter angesichts der Plötzlichkeit des Anschlags und seiner naturalistischen Wiedergabe erschrecken lässt, wird er bei 99 Problems durch die zuvor gezeigte latente wie präsente Gewalt vorbereitet und bekommt den Mord zudem in einer Weise präsentiert, die aufgrund ihrer starken (sich an die Schlussszene aus Arthur Penns Spielfilm Bonnie and Clyde (USA 1967) anlehnenden) Stilisierung auf den Symbolgehalt der vorgeführten Bilder hinweist. ${ }^{14}$ Dies bedeutet jedoch, dass die oben erwähnte Dokumentarästhetik des

12 So war man z. B. bei der zensierten Fassung von Spike Jonzes Video zu dem Stück Sabotage der Formation Beastie Boys vorgegangen: Da einzelne Momente der Bilderfolge als anstößig empfunden wurden (u. a. sah man dort, wie eine deutlich als solche erkennbare Puppe aus einem Auto bzw. von einer Brücke geworfen wurde), musste der Clip umgeschnitten werden, indem alternative Szenen nun die inkriminierten Stellen ersetzten. Vgl. dazu http://www.schnittberichte.com/index. php? ID =1137 [Stand: 12. 3. 2007]. Auf der Romanek-DVD (wie Anm. 9) berichtet Romanek demgegenüber, dass man die Musik von Jay-Zs 99 Problems sogar passagenweise habe umschneiden müssen, um der Erschießungsszene mehr Raum geben zu können.

13 Vgl. auch die Aussage Romaneks in einem am 24. 9. 2004 veröffentlichten Interview: „The ,bulletriddling' scene was really meant to be a kind of abstract, violent, ballet-like moment - a visual climax". Daher kann Romanek die Erschießungsszenen auch immer wieder mit Aufnahmen eines sich ekstatisch wiegenden Gospelchors sowie zur Musik tanzender Besucher eines Jay-Z-Konzerts durchsetzen. Zu dem ursprünglich auf http://www.mvwire.com/ veröffentlichten Interview vgl. http://www.markromanek.com/press/mvwirec.html [Stand: 12. 3. 2007].

14 In besagtem Film gerät das Gangsterpaar in einen Polizeihinterhalt und kommt in einem sie und ihr Auto förmlich durchsiebenden, zwanzigsekündigen Kugelhagel um. Penn greift hierbei bereits z. T. auf das Mittel der Zeitlupe und damit einer extremen zeitlichen Dehnung des dargestellten Moments zurück, wodurch der Tod der beiden Protagonisten ebenfalls die Züge einer grotesken Choreografie trägt. Die Möglichkeit dieser sehr detailgetreuen Darstellung verdankte sich u. a. dem Wegfall des sogenannten, zwischen 1930 und 1966 von der Filmindustrie sich selbst auferlegten „Production Code“, der zuvor geregelt hatte, was in Filmen hinsichtlich Sex und Gewalt dargestellt werden durfte. „Violence should shock“, wird Regisseur Arthur Penn zitiert, und Romuald Karmakar folgert im Begleittext einer DVD-Ausgabe von Penns Film im Oktober 2005 (ganz im Sinne von Romaneks „emotional impact“): „Nicht nur die Figuren, sondern auch die Zuschauer sollten ,getroffen' werden". Entsprechend empört und alarmiert reagierte die zeitgenössische Kritik auch auf Penns Darstellung des „Einschlag[s] einer Kugel in den menschlichen Körper [...] - parallel mit mehreren Kameras und unterschiedlichen Geschwindigkeiten gedreht“". Romaneks Entscheidung, diese Stilmittel auch für das Ende des Jay-Z-Clips zu verwenden, mag 


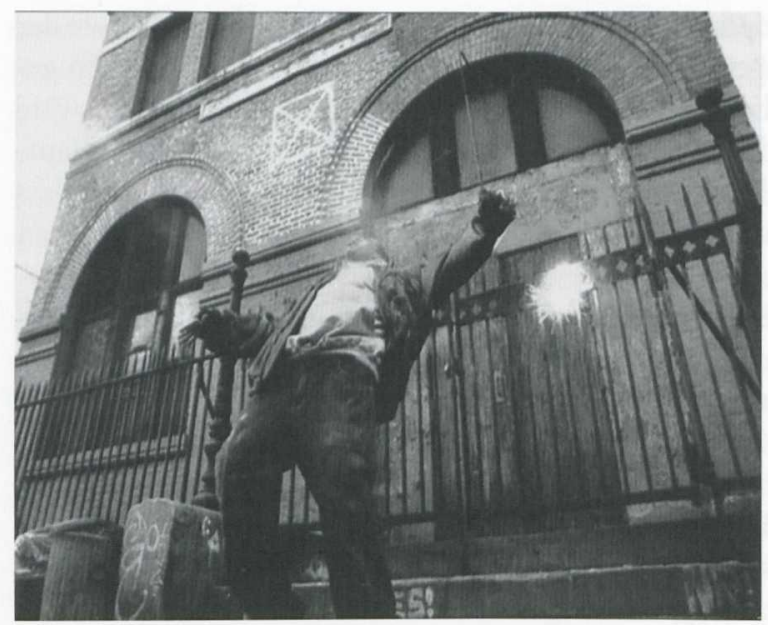

Abb. 1

Mark Romanek: Jay-Z, 99

Problems (Still), 2004. Aus:

The work of director Mark

Romanek. (C) 2005 Palm

Pictures. (DVD).

Clips nicht allzu sehr für bare Münze genommen werden darf beziehungsweise genauer geschaut werden muss, welchem übergeordneten Zweck sie hier dient.

Hierbei kann ein Vergleich mit einem der ,Ahnväter' des Musikvideos zu einem Rap-Song, Sylvia Robinsons Clip zu The Message von Grandmaster Flash and the Furious Five aus dem Jahr 1982, hilfreich sein. In dem Text des Stücks werden die sozialen Missstände, insbesondere im Alltagsleben der afroamerikanischen Bevölkerung angeprangert: Die Schilderung von schmutzigen und gefährlichen Wohnanlagen, Obdachlosigkeit, Verschuldung, Kriminalität und Zukunftslosigkeit mündet in die zentrale Formulierung: „You grow in the ghetto living second rate / And your eyes will sing a song of deep hate". Eben diesen fatalen Wirkzusammenhang von zweitklassigem Leben und daraus resultierendem (und so die Probleme perpetuierendem) Hass versucht der Interpret zu durchbrechen, indem er ihn offen legt; die Dringlichkeit einer Lösung unterstreicht er einmal mehr, wenn er in Bezugnahme auf sich selbst warnt: „Don't push me cause I'm close to the edge / I'm trying not to loose my head".

Regisseurin Sylvia Robinson gestaltete die visuelle Umsetzung des Songs, indem sie Grandmaster Flash and the Furious Five ihren Text an den dort genannten Schau-

- über die Suche nach einem geeigneten Verfahren, dessen Erschießung zu gestalten - auch durch den Umstand mitbestimmt sein, dass sich moderne Rapper oft und gerne als „Gangster" verstehen und daher auch mit Hilfe einer Ästhetik inszenieren, die auf die Verbrecherikonografie der Roaring Twenties zurückgeht; vgl. dazu z. B. Keazor, Henry; Wübbena, Thorsten, Video thrills the Radio Star-Musikvideos: Geschichte, Themen, Analysen, Bielefeld, 2007², S. 284. Zudem erwähnt Jay-Z selbst den Film in seinem Titel Song Cry von 2002 („Watchin Bonnie and Clyde, pretendin to be that shit") und interpretierte im gleichen Jahr den Song '03 Bonnie and Clyde zusammen mit seiner Lebensgefährtin Beyoncé Knowles, wobei beide sich mit den jeweiligen Geschlechterhälften des Gangsterpärchens identifizierten. Romanek bringt derartige Verweise auf frühere Karriereetappen der Interpreten gerne in seinen Videos unter - vgl. auch dazu Keazor; Wübbena, Video thrills the Radio Star, S. 355. 
plätzen - das Ghetto, dessen Straßen, Geschäfte und Abfallhalden - vortragen ließ; zwischen diese Performance-Sequenzen schnitt sie ferner (teilweise elektronisch verfremdete) Dokumentaraufnahmen, welche zu den im Stück erwähnten Szenarien passten: Schildert der Interpret dort zum Beispiel, dass sein Auto vom Gerichtsvollzieher beschlagnahmt wird, so rückt Robinson einen Abschleppwagen ins Bild; beschreibt der Text eine "Crazy lady livin” in a bag / Eating out of garbage piles", so zeigen die Bilder eine auf der Straße lebende, alte Frau. Doch obgleich die Regisseurin zum Ende des Clips sogar eine inszenierte Verhaftung der Interpreten durch eine Polizeistreife einbaut, um die im Song thematisierte Willkür und Ungerechtigkeit staatlicher Gewalt zu veranschaulichen, bleibt der Clip eigentümlich blass und ausdruckslos - gerade, wenn man ihn in Bezug zu der im Text artikulierten Empörung und Wut setzt. Robinson hatte, wohl auch um die Authentizität der verbal geschilderten Missstände zu unterstreichen, auf deren Bestätigung durch entsprechende Dokumentaraufnahmen gesetzt, damit jedoch eher eine bloße Illustration als ein gleichrangiges optisches Äquivalent zum Song von Grand Master Flash geliefert.

Demgegenüber arbeitet Mark Romanek bei 99 Problems auf eine emotionalisierende Gestaltung seines Clips hin, der den Betrachter vereinnahmen, packen und aufrütteln soll, und er bedient sich zu diesem Zweck einer freien Kombination aus Dokumentarästhetik und künstlerischer Stilisierung. Der Wunsch nach eben einer solchen Mischung soll es auch gewesen sein, der Jay-Z auf die Idee brachte, Romanek mit der Realisierung des Videos zu beauftragen: „I said, ,I want to shoot a pissy wall “", wird der Interpret in einem Interview anlässlich einer Vorpremiere des Videos am 23. April 2004 in New York zitiert: „You know how a photographer can make a pissy wall look like art? That's what I wanted. ${ }^{\text {“15 }}$ Mit der "pissy wall“ ist jedoch in diesem Fall nichts anderes als Brooklyn gemeint, und hierbei insbesondere die Wohnblocks der Marcy Projects Housing Community, einem ursprünglich dem Gedanken des sozialen Wohnungsbaus verpflichteten Vorhaben, das heute jedoch als ein von Schwarzen bewohntes Ghetto berüchtigt ist, in dem ein unbewaffneter Weißer angeblich nur wenige Sekunden Überlebenszeit hat.

Eben diese "uglier, bleaker side of Brooklyn" ${ }^{16}$ wollte Jay-Z in seinem Video dargestellt sehen, doch so, als ob er sich von dem oben erwähnten Clip zu Grandmaster Flashs The Message abzusetzen beabsichtigte, fügte er sogleich hinzu, dass er sich mit Romanek einen Regisseur gewünscht habe, der das Ganze „like art" fotografiere: „not shoot just a bunch of dudes or a bunch of cars around it - shoot it like art. And shoot it powerful and strong. " ${ }^{17}$

In dieser Idee einer Verschmelzung von Dokumentation (,the uglier, bleaker side of Brooklyn”) und Stilisierung (,shoot it like art [...] powerful and strong“") deckten sich die Vorstellungen von Interpret und Regisseur, und als Schnittstelle

15 Vgl. den ebenfalls von Shaheem Reid verfassten Artikel "Jay-Z gets blown away in provocative new video: ,99 problems ' debuts Sunday“, am 24. 4. 2004 veröffentlicht unter http://www.mtv.com/ news/articles/1486572/20040423/story.jhtml

16 Ebd.

17 Siehe Anm. 10. 
zwischen diesen beiden ästhetischen Sphären fungiert gerade das für den Clip gewählte Schwarzweiß. ${ }^{18}$ Es wird nicht von ungefähr auch von jenen Vertretern der sogenannten „New York School“ verwendet, deren Foto-Bücher Romanek während der Konzeptionsphase des Clips konsultierte: Ted Croner, Bruce Davidson, Helen Levitt, Weegee (Arthur Fellig) ${ }^{19}$ sowie Martin Dixon. ${ }^{20}$

Susan Sontag hat in ihrem 2003 erschienenen Buch Regarding the Pain of Others die Spannung diskutiert, welche Fotografien innewohnt, die schreckliche und leidvolle Dinge zeigen, diese jedoch mit einem anscheinend nicht objektiven, sondern stilisierenden Blick vorführen:

[...] people want the weight of witnessing without the taint of artistry, which is equated with insincerity or mere contrivance. Pictures of hellish events seem more authentic when they don't have the look that comes from being, properly' lighted and composed $[\ldots]$. By flying low, artistically speaking, such pictures are thought to be less manipulative - all widely distributed images of suffering now stand under that suspicion - and less likely to arouse facile compassion or identification. ${ }^{21}$

Indem die Fotografie nun aber zugleich als Dokumentationstechnik wie als künstlerisches Verfahren eingesetzt werden könne, werde sie mit einem ihr inhärenten, scheinbaren Widerspruch konfrontiert: „Photographs tend to transform, whatever

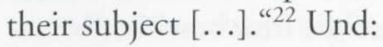

Transforming is what art does, but photography that bears witness to the calamitous and the reprehensible is much criticized if it seems, aesthetic; that is, too much like art. The dual powers of photography - to generate documents and to create works of visual art - have produced some remarkable exaggerations about what photographers

18 Sie erinnert zum einen an die Bilder eines Zeitungsberichts; zum anderen hatte sich - worauf schon Susan Sontag hinwies - auch ein Fotograf wie Henri Cartier-Bresson geweigert, mit Farbfilmen zu arbeiten: „In Cartier-Bresson's version of that persistent myth according to which - following the camera's invention - a division of territory took place between photography and painting, color belongs to painting." Sontag, Susan, „Photographic Evangels“, in: Dies., On Photography, New York, 1990 (erstmals erschienen 1977), S. 115-149, hier: S. 128 f. Vgl. dazu auch Roland Barthes, der die Farbe als eine Tünche oder Schminke bezeichnet, mit der die ursprüngliche Wahrheit des Schwarzweiß nachträglich zugedeckt werde. Barthes, Roland, La chambre claire, Paris, 1980, S. 128.

19 Zu ihnen vgl. Livingston, Jane, The New York School - Photographs 1936-1963, New York, 1992. $\mathrm{Zu}$ einer konkreten Rezeption der Fotos von Ted Croner siehe Anm. 34. In einem Interview mit Romanek, das Reto Caduff am 30. 8. 2004 unter der Überschrift „Reality-TV mit Seele“ in der Weltwoche (Ausgabe 35/04, unter „Kritik“) veröffentlichte, nennt Romanek ferner Eugene Richards. Das Interview kann auch im Internet unter http://www.weltwoche.ch/artikel/default. asp?AssetID=8571\&CategoryID=63 [Stand: 12. 3. 2007] nachgelesen werden.

20 Romanek hat dessen im Jahr 2000 in New York verlegten Fotoband Brooklyn Kings. New York City's Black Bikers Caduffs Angaben (wie Anm. 19) zufolge studiert; tatsächlich finden sich einzelne Einstellungen in 99 Problems, die bis in Details hinein wie in Bewegung versetzte Fotografien Dixons wirken (vgl. Abb. 5 a, b).

21 Sontag, Regarding the Pain of Others (wie Anm. 1), S. 27.

22 Sontag, Regarding the Pain of Others (wie Anm. 1), S. 76. Vgl. dazu auch Sontag, Regarding the Pain of Others (wie Anm. 1), S. 81: „Indeed, it is one of the functions of photography to improve the normal appearance of things." 
ought or not ought to do. Lately, the most common exaggeration is one that regards these powers as opposites. ${ }^{23}$

Romanek, der Kunstgeschichte studierte und die Überlegungen Sontags kennt ${ }^{24}$, verfolgte demgegenüber die Absicht, eben dieses „Doppelpotential“ der Fotografie auf den Videoclip zu übertragen und für eine Synergie zu nutzen, bei der die Stilisierung dem Dargestellten gerade seine visuelle Wucht verleiht, während umgekehrt die Dokumentarästhetik das Gezeigte durch den Eindruck von ,Echtheit noch präsenter und eindringlicher wirken lässt.

Auch einem an anderer Stelle von Sontag beschriebenen, weiteren Dilemma fotografischer Aufnahmen vermag Romanek mit der Form des Videoclips zu begegnen: In ihrem Aufsatz The Heroism of Vision stellt die Autorin fest, dass einem genuin politischen Kontext entstammende Fotos durch darin zugleich beobachtbare Anklänge an Werke der Malerei „depoliticized, for becoming a timeless image“25 würden. Indem das bewegte Bild des Clips die Assoziation an ein Gemälde zwar wecken, im nächsten Moment jedoch wieder konterkarieren kann, ist es möglich, der von Sontag beschriebenen Konsequenz entgegenzuarbeiten. Desgleichen können so die von ihr aufgeführten Gründe, weshalb in Filmszenen eingebaute Standfotos schockieren, gerade umgekehrt werden; Sontag schreibt, solche starren Bilder würden , $[\ldots]$ transmuting, in an instant, present into past, life into death “ ${ }^{26}$ Für in Filmsequenzen zitierte und sodann durch die Dynamik des bewegten Bildes wieder aufgelöste Arrangements aus Malerei und Fotografie könnte demgegenüber gelten, dass durch die einsetzende Verlebendigung "past into present“, „death into life“ rücküberführt werde.

Das Performative des Clips könnte mithin den Ausweg aus den von Sontag beschriebenen Aporien der Fotografie weisen, indem er die Bilder in Bewegung versetzt: Musikvideos zeigen eben nicht nur die „Performance“ eines Interpreten, sondern stellen selbst - dies kann anhand der von Dieter Mersch herausgearbeiteten Kategorien $^{27}$ und gerade im Vergleich mit Fotografien besonders gut aufgezeigt werden - einen performativen Akt dar.

23 Sontag, Regarding the Pain of Others (wie Anm. 1), S. 76.

24 In dem oben erwähnten Interview von Caduff (wie Anm. 19) verweist der Regisseur explizit auf die Bücher von Susan Sontag. Auf seiner Website (wie Anm. 8) berichtet Romanek unter „faq“: „later (sic!), i attended ithaca college, where i majored in cinema studies. However [...] my most worthwhile studies at ithaca were in the english, philosophy, art history, and religious studies departments."

25 Sontag, Susan, „The Heroism of Vision“, in: Dies., On Photography (wie Anm. 18), S. 83-112, hier: S. 105.

26 Sontag, Susan, „Melancholy Objects“, in: Dies.: On Photography (wie Anm. 18), S. 50-82, hier: S. 72 .

27 Mersch, Dieter, Ereignis und Aura. Untersuchungen zu einer Ästhetik des Performativen, Frankfurt am Main, 2002, S. $200 \mathrm{ff}$. Mersch betont S. 214 den Umstand, dass bei performativ konzipierten Kunstwerken „die ästhetische Erfahrung [...] sich [...] als Geschehen, als Prozess“ vollzieht und führt S. 219 auch dementsprechend Beispiele u. a. aus der Medienkunst an, die von eben diesem Prinzip der „Verzeitlichung“ (S. 225) lebt. Angesichts des Ereignischarakters, den die Ausstrahlung des Jay-Z-Clips durch seine Skandalträchtigkeit bekam, entspricht er in gewisser Weise auch der 
Die durch den Rekurs auf Fotografien erreichte Dokumentarästhetik des Videos passt überdies - und hieraus entwickelte Jay- $Z$ wohl seine damit konform gehende Konzeption - zum Gehalt des Songs 99 Problems mit seinen autobiografischen Rückverweisen.

Denn im Text (siehe Anhang) umreißt Jay- $Z$ drei zentrale Themen, die mit entsprechenden Schauplätzen assoziiert werden können. Im ersten Teil setzt er sich mit seinen Neidern, Kritikern und Gegnern auseinander, gegen deren vorschnelle und missgünstige Urteile er seine Herkunft aus sozial schwachen Verhältnissen in Anschlag bringt; werfen ihm diese zum Beispiel vor, dass er oberflächlich sei, da es ihm nur um Frauen und Geld gehe, so hält er dem seine ärmliche Jugend entgegen, aufgrund derer er Wohlstand durchaus zu schätzen wisse. Auch den Versuch, ihn zu Werbezwecken zu missbrauchen, kontert er mit dem Verweis auf seine Intelligenz und seine Erfahrungen, die es ihm überhaupt ermöglicht hätten, erst zu überleben und dann zu Reichtum zu kommen. Visualisiert wird diese Gegenüberstellung von ärmlicher Vergangenheit und begüterter Gegenwart durch Szenen, in denen der erwachsene Jay-Z am Wohnort seiner Kindheit und Jugend, eben den Marcy Projects, gezeigt wird.

In der zweiten Strophe von 99 Problems berichtet er von einer Episode aus der Zeit, als er sich als Drogenschmuggler finanzierte und dabei in eine Verkehrskontrolle geriet, die beinahe zu seiner Entdeckung und Verhaftung geführt hätte; das Video setzt zwar den Moment szenisch um, verzichtet aber auf die plumpe Nachinszenierung des im Lied in Form eines Melodrams gegebenen Dialogs zwischen den aggressiven Polizisten und dem sich selbstbewusst gebenden Protagonisten.

In der dritten und letzten Strophe entwickelt Jay- $Z$ eine Fantasie darüber, was passiert wäre, wenn ein Verleumder erfolgreich gewesen und ihn gemeinsam mit einem rassistischen Staatsanwalt ins Gefängnis gebracht hätte. Da er den Verräter im Text mit dem Slangausdruck "hoe" belegt (was sowohl eine saloppe Anrede unter Männern wie auch ein despektierlicher Begriff für eine Frau, zum Beispiel eine Prostituierte, sein kann) ${ }^{28}$, ist dies für Romanek der Anlass, Szenen mit Prostituierten und ihrem Zuhälter zu zeigen, während die dazwischen geschnittenen Aufnahmen eines Leichenbestatters auf Liedzeilen reagieren, in denen das lyrische Ich für den Verräter betet („I tried to ignore him and talk to the Lord / Pray for him, cause some fools just love to perform" - dazu hält die Kamera auf das Kruzifix, das über einem Sarg hängt). Mit diesen Bildern hat der Clip dann auch endgültig in die Todesthematik eingeschwenkt, die im Folgenden mit der Ermordung Jay-Zs zu ihrem Höhepunkt und Abschluss kommt. Latent präsent war dieses Mo-

von Mersch S. 218 getroffenen Feststellung: „Die Ästhetik des Performativen markiert so einen ,Sprung' ins Andere, den Sprung vom, Werk' zum ,Ereignis'."

28 Vgl. dazu z. B. das Online Slang Dictionary unter http://www.ocf.berkeley.edu/-wrader/slang/h. html [Stand: 12. 3. 2007]: , hoe $=1$. a greeting, usually between males. (,What's up, hoe?") n 1. diminutive form of , whore. 2. an unfriendly person, usually a female. (,She is such a hoe.") ". Eben die Etymologie des Wortes „hoe“ (also von „Hure” herstammend) thematisiert Jay-Z in seinem Text, wenn er klar stellt, dass er damit keine Frau meine („This is not a hoe in the sense of havin' a pussy“), sondern einen schwächlichen Feigling („But a pussy havin’ no God Damn sense [...]"). 
tiv jedoch schon von Anfang an, wenn der Interpret gleich in den ersten zwei Zeilen seines Stückes davon berichtet, dass er von feindlichen Rappern verfolgt wird, die ihn töten wollen: „I got the rap patrol on the gat patrol / Foes that wanna make sure my casket's closed“. Auch der am Ende der Strophen wiederholt zum Refrain überleitende Ausruf „Hit me!“ (eigentlich ein Slangausdruck für die Anweisung „Play it!", mit welcher der Kehrreim eröffnet werden soll) deutet - wörtlich als "Triff mich!“" verstanden - auf das spätere Attentat voraus, wo dieser Aufforderung auf brutale Weise nachgekommen wird. Anfang, Verlauf und Ende des Videos sind so in Text und Bild eng miteinander verflochten.

Doch mit den Szenen beim Leichenbestatter wird zugleich ein weiteres Thema offenkundig gemacht, das die Bilderfolge zuvor bereits latent durchzogen hat: Das der Religion beziehungsweise der verschiedenen Religionen. Denn da im „Melting Pot" Brooklyn unterschiedliche Ethnien zusammenleben, rückt der Clip, auch um den Stadtteil adäquat zu charakterisieren, immer wieder Anzeichen der Glaubensvielfalt in den Blick. „I'm living for Jesus - How about you?" fragt beispielsweise gleich zu Beginn ein Aufkleber über dem Spion der Tür, die in eine eingangs gezeigte Wohnung der Marcy Houses führt; kurz darauf hält die Kamera zunächst auf einen orthodoxen Juden, weniger als eine Minute später dann auf das Kruzifix zu. Dazwischen geschnitten finden sich Aufnahmen einer Konfuzius-Statue und eines in ähnlicher Haltung Meditierenden (Abb. 2 a, b); er scheint Jay-Zs Tod in einer Art Vision wahrzunehmen, denn er reißt die zur inneren Schau geschlossenen Augen weit auf, nachdem die ersten Kugeln den Rapper getroffen haben, und schlägt sich sodann voller Entsetzen die Hände vor das Gesicht - die Reaktion des Zuschauers somit gleichsam idealtypisch vorwegnehmend (Abb. 2 c-e). ${ }^{29}$

Auf Elemente religiöser Ikonographie wird jedoch ebenfalls im Rahmen eines besonders zum Auftakt des Clips eingesetzten Verfahrens angespielt, das auf die Verunsicherung des Betrachters abzielt. So wird dieser von einem jungen Mädchen angeblickt, das in seiner symmetrischen Frontalität, mit seinem mutmaßlich von einem Tuch verhüllten und so das Gesicht betonenden Kopf (Abb. 3 a) etwas von den Madonnenbildern eines Antonello da Messina zu haben scheint (Abb. 3 b) ${ }^{30}$ - doch im nächsten Augenblick wird dieses Arrangement (damit die oben angesprochene Aporie Sontags auflösend) gleichsam gesprengt, wenn das Mädchen die Umhüllung mit weit ausgestreckten Armen von sich wirft und sie so zugleich als simple Bettdecke ausweist (Abb. 3 c).

29 Diese Reaktion wird sodann von derjenigen des Staatsanwaltes sekundiert und konterkariert, der in einer Geste äußerster Hilflosigkeit seinen Aktenkoffer gegen einen Zaun schleudert. Reid (wie Anm. 15) berichtet von der Uraufführung des Clips, dass die Erschießungsszene „drew a gasp from some members of the audience".

30 Vgl. dazu z. B. auch Sontag, Regarding the Pain of Others (wie Anm. 1), S. 80: „To feel the pulse of Christian iconography in certain wartime or disaster-time photographs is not a sentimental projection. " Zu Antonellos Gemälden mit der Madonna der Verkündigung (Palermo, Galleria Regionale della Sicilia: datiert auf 1476/77 sowie München, Alte Pinakothek: datiert auf 1473/74) vgl. u. a. Barbera, Gioacchino, Antonello da Messina, Mailand, 1998, S. 63 (München) und S. 134 (Palermo) sowie Savettieri, Chiara, Antonello da Messina, Palermo, 1998, S. 69 f., No. 4 (München) und S. 70 f., No. 5 (Palermo). 

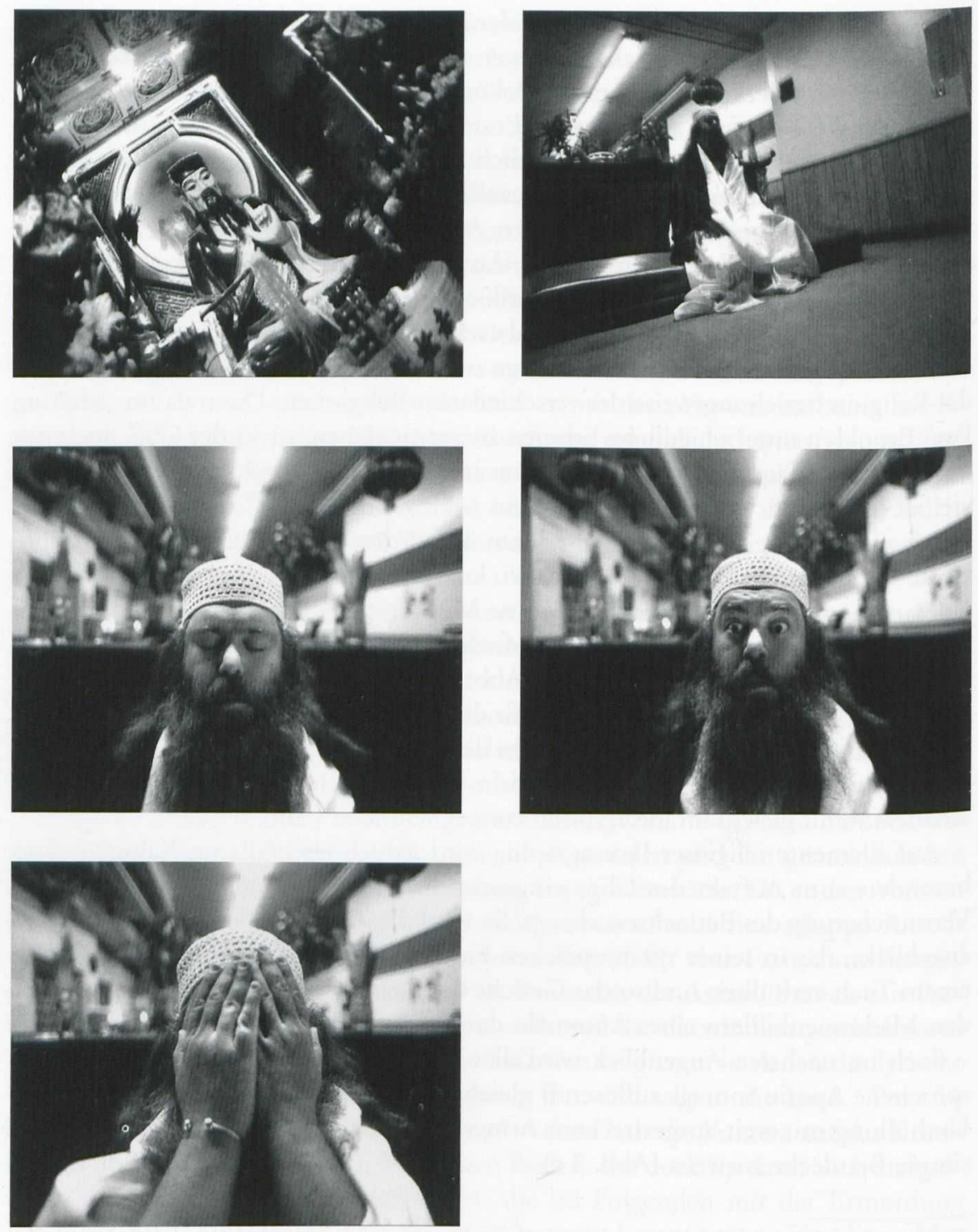

Abb. 2 a-e

Mark Romanek: Jay-Z, 99 Problems (Stills), 2004. Aus: The work of director Mark Romanek. (C) 2005 Palm Pictures. (DVD). 

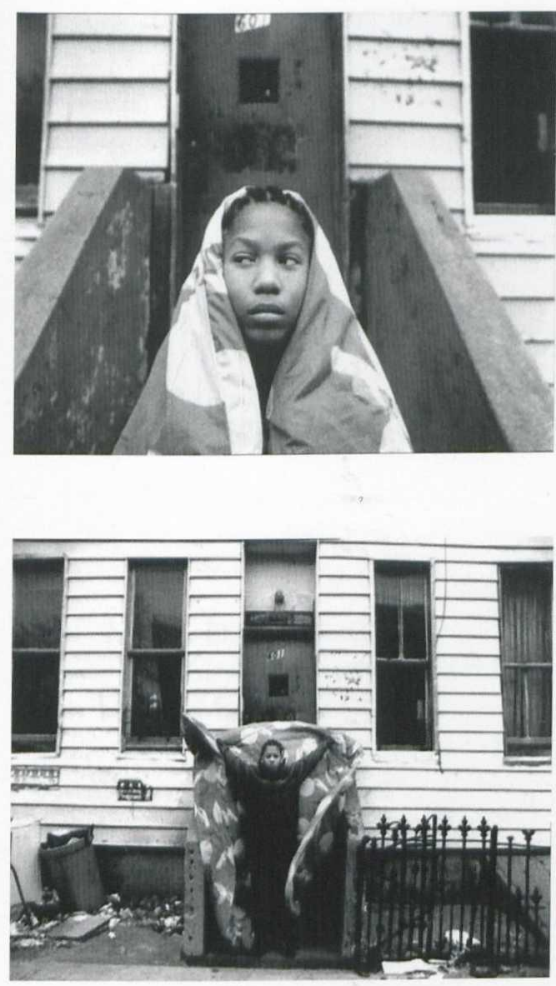

Abb. 3 a, c

Mark Romanek: Jay-Z, 99 Problems

(Stills), 2004. Aus: The work of director

Mark Romanek. (C) 2005 Palm Pictures.

(DVD).

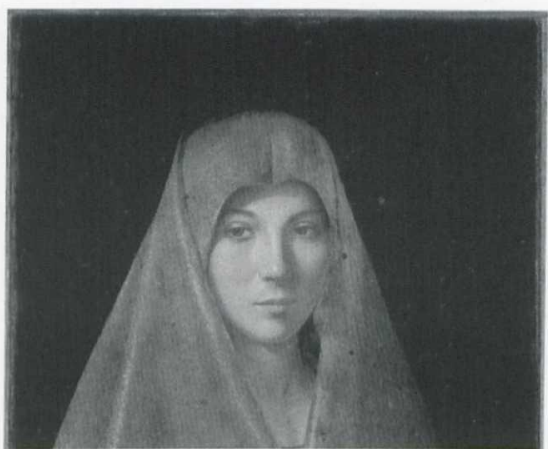

Abb. 3 b

Antonello da Messina: Madonna der Verkündigung, Detail, (Palermo, Galleria Regionale della Sicilia), 1476/77. Aus: Fiorella Sricchia Santoro, Antonello e l'Europa, Mailand, 1986, Abb. 37.

In ähnlicher Weise, nun jedoch bar jeglichen religiösen Kontextes, funktionieren Szenen, in denen weitere Menschen in die Kamera blicken: „In the normal rhetoric of the photographic portrait, facing the camera signifies solemnity, frankness, the disclosure of the subject's essence", schreibt Susan Sontag. ${ }^{31}$ Tatsächlich schaut uns einmal ein Junge mit großen, weit geöffneten Augen (Abb. 4 a), ein anderes Mal ein die Mundpartie in seiner Jacke vergrabender Mann an, die beide traurig, schutzlos und verletzlich wirken. Der Mann hält der Kamera zudem scheinbar werbend ein großes Stoffherz mit der Aufschrift "Love“ entgegen. Doch im nächsten Moment erweist es sich, dass wir es mit Personen zu tun haben, die sich

31 Sontag, Susan, „America, Seen Through Photographs, Darkly“, in: Dies., On Photography (wie Anm. 18), S. 25-48, hier: S. 38. 

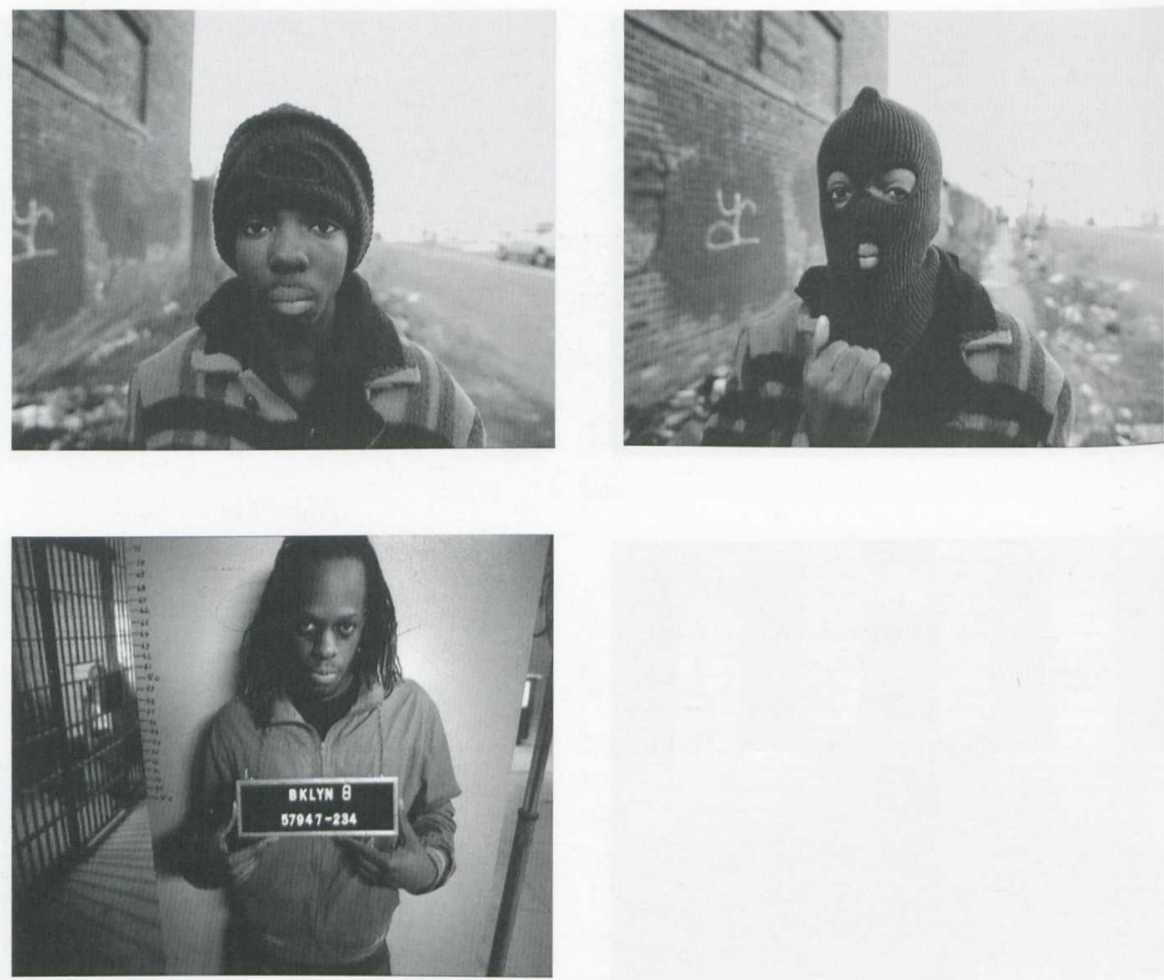

Abb. 4 a-c

Mark Romanek: Jay-Z, 99 Problems (Stills), 2004. Aus: The work of director Mark Romanek. (C) 2005 Palm Pictures. (DVD).

gegenüber „the camera's aggressions“ ${ }^{32}$ durchaus zu behaupten wissen. Nichts ist wie es zunächst scheint, denn vermeintlich Harmloses schlägt im nächsten Moment in Bedrohliches um: Der Mann zerknüllt das Stoffherz rücksichtslos und wirft es dem Betrachter entgegen; der Junge streift eine Wollmütze über, die sich mit ihren ausgeschnittenen Mund- und Augenlöchern als zu der Art Maske präpariert erweist, wie sie bei Raubüberfällen getragen wird (Abb. 4 b). Nicht umsonst kehren die dem Betrachter frontal zugewandten Gesichter dann auch zu einem späteren Zeitpunkt des Clips wieder, wenn gefasste Verbrecher erkennungsdienstlich fotografiert werden (Abb. 4 c) und der Zuschauerblick zugleich derjenige des Polizeifotografen zu sein scheint: Fotografie und Film, statische Aufnahme und bewegtes Bild fallen in diesem Moment in eins. Schon zuvor hat die hohe Frequenz, mit der sich die Szenen gegenseitig durchdringen, dafür gesorgt, dass die kurzen Fragmente zuweilen den Charakter von bewegten Fotografien annehmen,

32 Sontag, „The Heroism of Vision“ (wie Anm. 25), S. 104. 
Abb. 5 a

Mark Romanek: Jay-Z, 99 Problems (Still), 2004. Aus: The work of director Mark Romanek. (C) 2005 Palm Pictures.

(DVD).

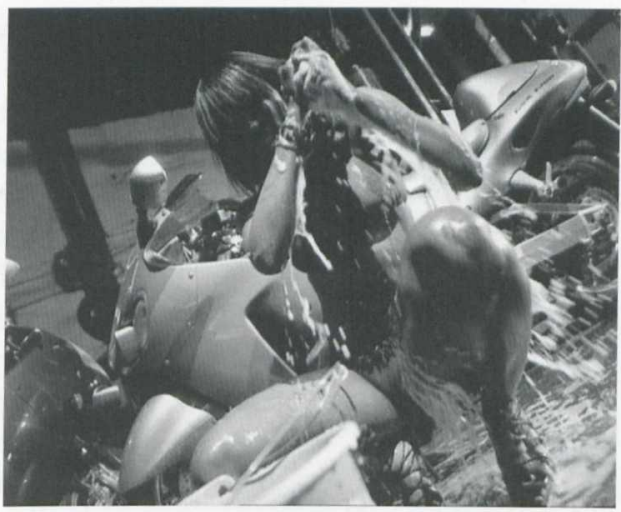

Abb. 5 b

Martin Dixon: Brooklyn Kings. New York City's Black Bikers, 2000. Aus:

Dixon, Martin, Brooklyn Kings. New York City's Black Bikers, New York, 2000, S. 56, 57.

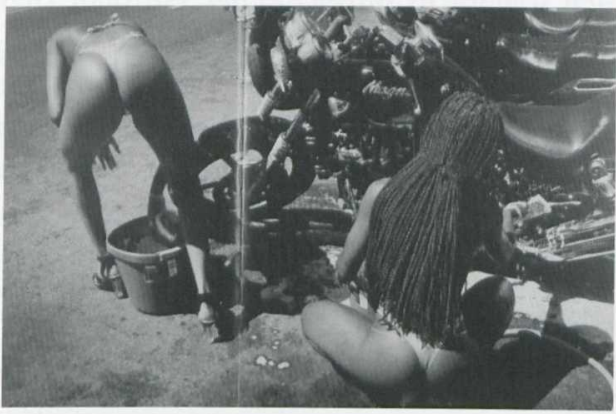

da sie nicht lange genug andauern, um sogleich eine Handlung nachvollziehbar werden zu lassen. Die einzelnen Szenen der verschiedenen Kontexte sind vielmehr so in- und gegeneinander geschnitten, überholen und antizipieren, durch- und überkreuzen sich dergestalt, dass sich - wie oben anhand der religiösen Thematik gesehen - die einzelnen Motivbereiche, obgleich früh durch Bilder vertreten, zuweilen erst sehr viel später entfalten, um dann vorübergehend ganz in den Vordergrund zu treten: So erscheinen die durch Martin Dixons Bildband Brooklyn Kings inspirierten Sequenzen (Abb. 5 a, b) ${ }^{33}$ bereits während der zweiten Strophe, obgleich sie streng genommen ihr textliches Äquivalent erst im dritten Teil von 99 Problems haben, wo die Metapher „loud as a motorbike" fällt; auch die Prostituierten und ihr Zuhälter sind zu Beginn des Clips bereits kurz zu sehen, obgleich der Zuschauer eigentlich erst im letzten Drittel des Videos erfährt, um wen es sich bei den Auftretenden handelt. ${ }^{34}$

33 Vgl. dazu Anm. 20.

34 Insofern ist auch der chronologische Ablauf der einzelnen Vorgänge gesprengt, was die Eindrücklichkeit sowohl der einzelnen Sequenzen als auch von deren Aufeinandertreffen im sorgfältig kalkulierten Gefüge der Schnitte erklärt, denn diese gehorchen einem Prinzip, das Susan Sontag als 
Der Clip destabilisiert jedoch auch insofern Gewissheiten, als er die typischerweise angesichts eines Rap-Videos gehegten Erwartungen weckt und im nächsten Moment wieder enttäuscht. „HipHop ist ein Genre, das mit einem extremen Macker-Posing daherkommt und Frauen am liebsten im Bikini sieht", charakterisierte Heike Blümner 1999 die Gattung stark verknappt ${ }^{35}$, doch treffend. Eben diese Bikini-Frauen sowie der in Rap-Videos ebenfalls obligate Goldschmuck tauchen in 99 Problems zwar auf, werden dabei jedoch in einer eigenwilligen Brechung vorgeführt, wenn der Schmuck lediglich in Vitrinen ausliegt, anstatt vom Interpreten getragen zu werden, und die Bikini-Frauen nicht, wie sonst üblich, gemeinsam mit dem Schmuck als Statussymbole des Protagonisten vorgeführt, sondern unter anderem mit dessen Erschießung gegeneinander geschnitten werden.

Derartige Abweichungen von der gewohnten und stereotypen Faktur herkömmlicher Rap-Videos passen insofern gut zu dem in Rede stehenden Stück, als dieses sich sowohl in musikalischer Hinsicht (nämlich mit seinen der Rockmusik entlehnten und hier als Träger des Sprechgesangs fungierenden, ostinat wiederholten, aggressiven Gitarrenakkorden) als auch textlich explizit von der Vielzahl all jener Dutzend-Songs abgrenzt, in denen es lediglich um Liebesprobleme mit Frauen geht: „If you're havin' girl problems I feel bad for you, son / I got 99 problems but a bitch ain't one" ${ }^{36}$ eröffnet Jay- $Z$ seine Schilderungen.

Über diese und andere Bezüge des Clips zu dem einzelnen Song weist Romaneks Schöpfung jedoch zugleich in vielerlei Hinsicht Rückbindungen an die im November 2003 erschienene CD The Black Album auf, aus der 99 Problems fünf Monate später als Single ausgekoppelt wurde. Ist das Video sicherlich auch in Schwarzweiß gehalten, um die Authentizität der gezeigten Bilder und damit Jay-Zs Verankerung in der Kultur des afroamerikanischen Brooklyn (mithin seine so genannte „Street Credibility") zu belegen, so scheint diese Ästhetik doch zugleich ein Echo auf die

typisch für die Fotografie bezeichnet hat: „Photographs may be more memorable than moving images, because they are a neat slice of time, not a flow“. Sontag, Susan, „In Plato's Cave“, in: Dies., On Photography (wie Anm. 18), S. 3-26, hier: S. 17. Eben das Aufsprengen eines solchen Ablaufs nimmt dem Betrachter von 99 Problems zudem die Gewissheit, dass Jay- $Z$ am Ende des Clips doch noch am Leben sein könnte: Zwar endet das Video damit, dass ein grinsender Jay-Z der Kamera einen Faustschlag verpasst, wodurch diese das Bild der im Abendlicht liegenden Marcy Houses wie von einem heftigen Erdbeben erschüttert aufnimmt (das entstehende Bild wirkt wie eine in Bewegung versetzte Fotografie Ted Croners, z. B. dessen Untitled, New York von 1947/50. Siehe Abb. 7 c, d. Zu den Arbeiten von Croner siehe: Livingston, The New York School (wie Anm. 19), S. 258 ff.); da jedoch zuvor schon eine chronologische Abfolge der Szenen konsequent gestört wurde, sagt dies nichts über den tatsächlichen zeitlichen Kontext dieser letzten Szene.

35 Blümner, Heike, „Street Credibility. Hip-Hop und Rap“, in: Alles so schön bunt hier. Die Geschichte der Popkultur von den Fünfzigern bis heute, hg. von Peter Kemper, Thomas Langhoff, Ulrich Sonnenschein, Stuttgart, 1999, S. 254-265, hier: S. 260.

36 „Bitch“ ist hier - ähnlich wie „hoe“ (siehe Anm. 28) - durchaus nicht ausschließlich negativ konnotiert; das Online Slang Dictionary (wie Anm. 28) gibt dazu an, dass der Begriff (wörtlich eigentlich „Hure") ,in some groups, however, [...] is used as a well-meaning greeting"; im vorliegenden Fall (vgl. auch Anm. 28) stellt er einen Slangausdruck für „Frau“ dar. 
Abb. 6 a

Mark Romanek: Jay-Z, 99 Problems (Still), 2004. Aus: The work of director Mark Romanek. (C) 2005 Palm Pictures. (DVD).

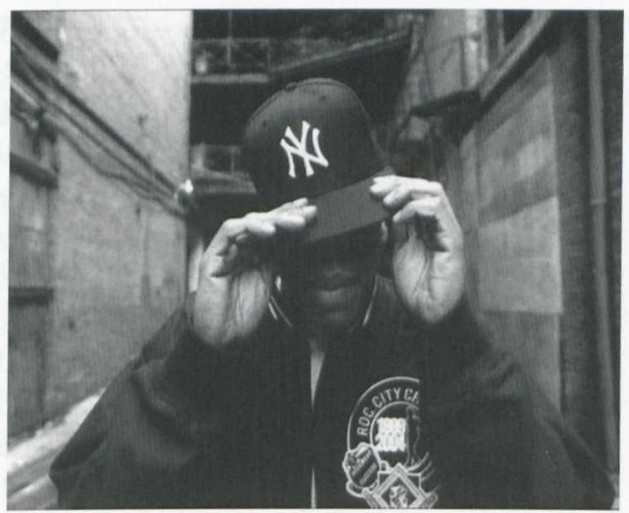

Abb. 6 b

Robert Sims: Jay-Z, The Black Album, (Cover des Booklets), 2003. (C) 2003

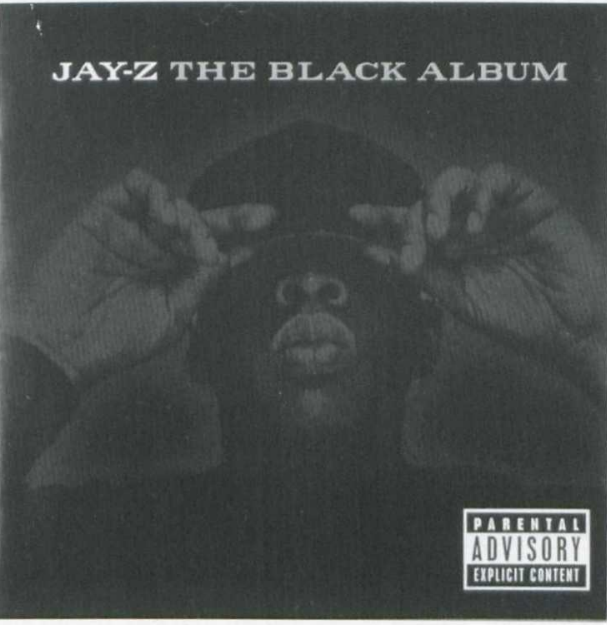

Roc-A-Fella Records.

ebenfalls in strengem Schwarzweiß gehaltene Verpackung der CD darzustellen. ${ }^{37}$ Darüber hinaus wirken einige Szenen aus dem Clip (Abb. 6 a), als ob aus ihnen zum Beispiel die (tatsächlich sehr viel früher fotografierte) Coverillustration des Booklets gewonnen sei, das dem Album beiliegt (Abb. 6 b). Auf der dort gezeigten Aufnahme hebt sich das extrem dunkel gehaltene Portrait Jay-Zs schattenhaft gerade noch von dem schwarzen Untergrund ab, damit nicht nur dem Titel der CD The Black Album - bildlichen Ausdruck verleihend, sondern zugleich eine Zeile visualisierend, die zum Schluss des darauf befindlichen Stücks December 4 th wiederholt wird: „Maybe you love me when I fade to black“. Das hier angesprochene

37 Die Verpackung besteht aus einer schwarzen CD-Plastikhülle, auf deren Vorder- und Rückseite lediglich schwarzweiße Aufkleber mit dem Titel sowie der Bestellnummer und dem Copyright angebracht sind. 
Schwarz steht dabei natürlich - wie bei der Wahl der Verpackungsgestaltung - für den Tod: The Black Album bezeichnete den Rückzug Jay-Zs aus dem Musikgeschäft und den Abschied Shawn Carters von seiner Rap-Existenz als Jay-Z..$^{38}$ Diese Stellung der $\mathrm{CD}$ innerhalb von dessen Oeuvre prägt dann auch viele Texte der dort versammelten Stücke: „All things must come to an end“, heißt es auf dem das Album eröffnenden Interlude. "We see what happens when I no longer exist", droht der Interpret auf What More Can I Say, und December 4th erweist sich sogar als eine Art von Kurzbiografie, denn zu den Klängen eines Samples aus dem Song That's How Long von $1974^{39}$ berichtet die Mutter des Interpreten, Gloria Carter, von der Geburt, ${ }^{40}$ Kindheit und schwierigen Jugend ihres Sohnes, der darauf ankündigt: "They say they never really miss you / Till you're dead or you're gone / So on that note I'm leaving after this song“, um sich am Schluss mit eben den Worten „Maybe you love me when I fade to black" aus dem Stück zu verabschieden. Die in 99 Problems geführte Auseinandersetzung mit Neidern, Kritikern, Feinden sowie der eigenen Vergangenheit als Drogenhändler gliedert sich so als ein Bestandteil in die auf dem Black Album vorgelegte biografische Rückschau ein.

Romaneks Idee, Shawn Carters Rap-Ego Jay-Z zum Ende des zugehörigen Clips den Kugeltod sterben zu lassen, erweist sich angesichts des auf dem Album in Gestaltung und Texten präsenten Themenkomplexes von Tod, Verlust und Liebe als konsequenter Versuch, den Grundgestus der CD in dramatisierte Bilder zu gießen - zumal auf den dort ebenfalls versammelten Stücken Threat und Allure Schüsse zu hören sind. ${ }^{41}$

Die so erreichte Verzahnung des Videos mit den Themen und der Ästhetik des Albums, dem 99 Problems entstammt und dessen Kontext erst durch die Bilderfolge Romaneks an den Song herangetragen wird, fällt hier dichter aus, als etwa in dem Clip, den der Regisseur sieben Jahre zuvor für Janet Jacksons Stück Got 'Til It's Gone gedreht hatte. ${ }^{42}$ Da das dem 1997 veröffentlichten Song zugrunde liegende Sample aus dem 1970 eingespielten Lied Big Yellow Taxi von Joni Mitchell stammt ${ }^{43}$, ver-

38 Zur tatsächlichen Dauer dieses Rückzugs vgl. Anm. 3. Mit der Titelwahl The Black Album brachte Jay-Z sich zugleich in einen bewussten Kontrast zum 1968 veröffentlichten White Album der Beatles; dass diese Gegensätzlichkeit verstanden wurde, zeigt das Unternehmen des Musikers DJ Dangermouse, der im Februar 2004 eine Platte vorlegte, auf dem die Stimme von Jay-Zs schwarzem und die Tracks vom weißen Album der Beatles zum Grey Album abgemischt wurden. Die Vocals von 99 Problems werden hier z. B. von Klängen aus Helter Skelter begleitet.

39 Der Song stammt von der Formation The Chi-Lites. Vgl. dazu: http://www.soultracks.com/chilites.htm [Stand: 12. 3. 2007].

40 Shawn Carter wurde am 4. 12. 1969 geboren. Die von seiner Mutter berichteten Umstände folgen dem klassischen Schema der Geburt eines Wunderkindes: „Weighing in at 10 pounds 8 ounces, he was the last of my four children, the only one who didn't give me any pain when I gave birth to him. And that's how I knew that he was a special child."

$41 \mathrm{Zu}$ einem ähnlichen Verfahren Romaneks bei Michael Jacksons Song Scream vgl. Keazor; Wübbena, Video thrills the Radio Star (wie Anm. 14), S. $344 \mathrm{ff}$.

42 Das Video wurde im August 1997 veröffentlicht, um das unmittelbar danach - im September 1997 - auf den Markt gebrachte Album The Velvet Rope zu bewerben.

43 Veröffentlicht auf dem im April 1970 erschienen Album Ladies of the Canyon. Vgl. dazu u. a. http://www.jonimitchell.com/HMLadies70.html [Stand: 12.3. 2007]. 
suchte Romanek, diesen Vergangenheitsbezug dadurch zu thematisieren, dass er die (bereits hier wie zum Teil belebte Fotografien konzipierten) Bilder des Clips in einem Setting ansiedelte, das sich an die Mode und Ästhetik einer weiter zurückliegenden Zeit anlehnte. Zwischen den Liedern von Janet Jackson und Joni Mitchell bestand über das musikalische Zitat hinaus inhaltlich kein engerer Bezug ${ }^{44}$, was den Regisseur offenbar davon dispensierte, die Interpretin zwingend in einem Siebziger-JahreAmbiente zu inszenieren. ${ }^{45}$ Stattdessen griff er die Mitte der neunziger Jahre wieder modische Kultur der „Shebeens“, den „Social Halls“ auf, die im Südafrika der fünfziger Jahre die schwarze Bevölkerung gründete und frequentierte. ${ }^{46} \mathrm{Da}$ diese mit ihrem Gemisch unterschiedlicher, sich vor allem bei der amerikanischen Gangsterkultur der zwanziger Jahre inspirierender Stile auch wesentlich musikalisch geprägt war, schien dieses (zudem leicht morbid erscheinende) Ambiente der geeignete Rahmen für eine Inszenierung von Janet Jacksons melancholischem Lied.

In 99 Problems hingegen hat Romanek dafür gesorgt, dass die musikalische Faktur des Liedes direkter im Clip präsent ist, denn der (von Jay-Z gegen Ende des Stücks auch adressierte) Produzent Rick Rubin, der die beiden dem Song zugrunde liegenden Samples zusammengestellt hat, spielt in dem Video selbst mit (und ist dort daher unter anderem zu sehen, wie er in einem Laden Schallplatten aussucht). ${ }^{47}$ Er tritt zum einen als Jay-Zs Beifahrer auf (wie in der Szene der Verkehrskontrolle, die damit endgültig von einem etwaigen Versuch der getreuen Nachinszenierung abgesetzt wird, da Interpret und Produzent sich damals noch nicht kannten); zum anderen jedoch sieht man ihn immer wieder in Begleitung des Schauspielers Vincent Gallo (Abb. 7 a). Über die Gründe für dessen Beteiligung an dem Video ist wiederholt gerätselt worden ${ }^{48}$, da er scheinbar keinerlei direkte Beziehung zu den

44 Bei Mitchells Song handelt es sich um ein sozialkritisches und politisch engagiertes Stück, das gegen die Naturzerstörung plädiert. Janet Jackson griff sich den Refrain „Don't it always seem to go / That you don't know what you've got / Till it's gone“ und baute daraus ein Lied um eine gescheiterte Liebesbeziehung, in der sich die Frau eben diesen Satz nun immer wieder vorsagt, um sich in Reue an den Mann zu erinnern, den sie hat gehen lassen.

45 Er zeigt Janet Jackson nur einmal, wie sie neben einem Fernseher steht, auf dem ein Filmausschnitt mit Joni Mitchell zu sehen ist. Vgl. dazu auch Großmann, Rolf, „Xtended Sampling“, in: Sampling. Arbeitsberichte der Lehrkanzel für Kommunikationstheorie, Heft 4, hg. von Hans-Ulrich Reck, Mathias Fuchs, Hochschule für angewandte Kunst, Wien, 1995, S. 38-43 sowie als Xtended Sampling II - HTML Statements unter: http://www.audio.uni-lueneburg.de/webseiten/xsamp/ xsamtext.htm [Stand: 12. 3. 2007].

$46 \mathrm{Zu}$ diesen und ihrer Verwendung in der Modewelt der neunziger Jahre vgl. http://www.designindabamag.com/2002/1st/artical02-what.html [Stand: 12. 3. 2007].

$47 \mathrm{Zu}$ ihm vgl. u. a. http://www.mtv.com/bands/r/rubin_rick/news_feature_040503/[Stand: 12. 3. 2007].

48 Vgl. dazu z. B. Rotter, Jeffrey, "Jay-Z wants to kill himself“, in: The New York Times, 9. 5. 2004, Arts and Leisure Desk, Section 2, S. 13: „The song's producer, Rick Rubin, and - inexplicably the actor Vincent Gallo make morose cameos.“ Angesichts des Nachdrucks, mit dem die Gründe für dessen Anwesenheit in dem Clip von den Beteiligten im Nachhinein herunter gespielt wurden, erscheint diese umso interessanter: Rubin erzählt im Rahmen des „Making of 99 Problems“ auf der oben erwähnten DVD (wie Anm. 9), dass Gallo angeblich mehr oder weniger zufällig zu den Dreharbeiten hinzu gestoßen sei; Romanek selbst behauptet dort, dass es tatsächlich keinen Grund für dessen Mitwirkung gegeben habe - er sei eben „one of the most photogenic humans in the 


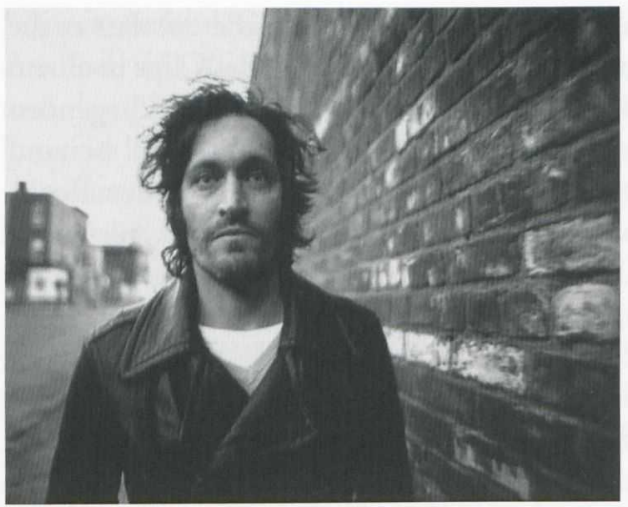

Abb. 7 a

Mark Romanek: Jay-Z, 99 Problems

(Still: Vincent Gallo), 2004. Aus: The

work of director Mark Romanek. (C) 2005

Palm Pictures. (DVD).

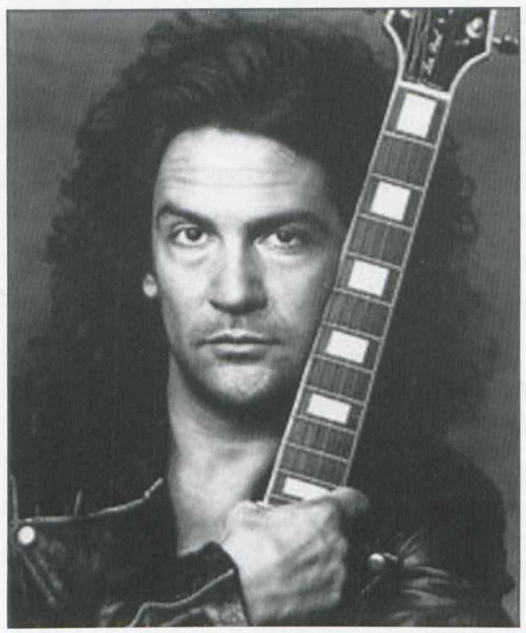

Abb. 7 b

Richard Noble: Billy Squier, 1989 (für das Album Hear and Now). (C) 1989 Capitol Records.

Musikern und ihrem Stück hat. ${ }^{49}$ Tatsächlich jedoch tritt Gallo nicht als er selbst auf $^{50}$, sondern soll hier offenbar den Gitarristen Billy Squier verkörpern, aus des-

world. "Andererseits wird Romanek von allen Künstlern, für die er Videos drehte, für die Präzision der von ihm von Anfang an verfolgten Vorstellungen gelobt, so dass eine derartige Zufälligkeit wenig glaubwürdig erscheint.

49 Auf der Website http://www.popzoot.tv/streams.php3?video=994\&rubrik=4e [Stand: 12. 3. 2007] wird zurecht darauf hingewiesen, dass der auch als Maler, Komponist, Regisseur und Produzent tätige Gallo durch seine Mitwirkung in Filmen über die Hip-Hop-Kultur eine entsprechende Vergangenheit hat; allerdings wäre dies noch immer ein nur sehr lockerer Bezug, der angesichts des ansonsten so dichten motivischen Gefüges des Clips überraschen würde. Zu Gallos vielseitigen Aktivitäten und seiner schillernden Persönlichkeit vgl. Bottero, Giuseppe; Lombardo, Marco, I Cattivi - Vincent Gallo. Bassa fedeltà, Mailand, 2006.

50 Es wurde Romaneks Clip jedoch offenbar zum Problem, dass Gallo als Person aus der Musik- und Filmwelt selbst zu prominent ist, um einen anderen Musiker verkörpern zu können, so dass er hier als nur für sich selbst stehend und nicht als Schauspieler einer Rolle erkannt wurde. 
Abb. 7 c

Mark Romanek: Jay-Z, 99 Problems (Still), 2004. Aus: The work of director Mark Romanek. (C) 2005 Palm Pictures.

(DVD).

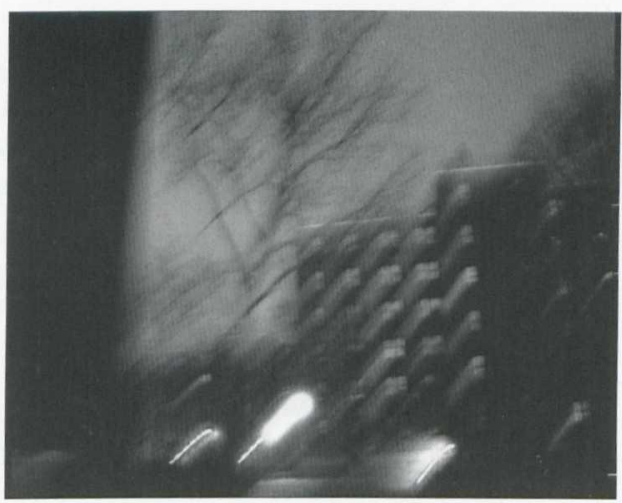

Abb. $7 \mathrm{~d}$

Ted Croner: Untitled, New York, 1947-50. Aus: Jane Livingston, The New York School - Photographs 19361963, New York, 1992, S. 257.

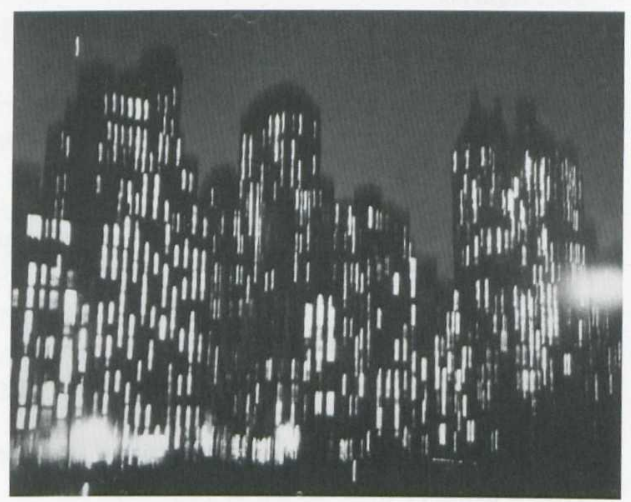

sen Stück The Big Beat ${ }^{51}$ Rubin das musikalische Grundmaterial zu 99 Problems gewonnen hat. Im "Look“ des Billy Squier der achtziger Jahre, nachdem er Big Beat aufgenommen hatte (Abb. 7 b), stürmt Gallo zusammen mit Rubin durch die Straßen von Brooklyn, begleitet von den durch sie geschaffenen, ostinaten Klängen: dem treibenden Schlagzeugmotiv und den aggressiv vorwärts drängenden Gitarrenakkorden, zwischen denen immer wieder ein Aufschrei ertönt. ${ }^{52}$ Diese Musik wird nicht nur in das dichte, mit Romaneks Clip gewobene Geflecht aus Text, Klängen und Bildern eingearbeitet, sondern hat zudem selbst einen nicht unwesentlichen Anteil an dem angestrebten „unforgettable emotional impact“.

51 Von dem 1980 erschienenen Album The Tale of the Tape.

52 Dies ist, ebenso wie der pochende, synkopierte Rhythmus, ein Zitat aus dem Stück Long Red der Gruppe Mountain. Das Stück stammt von Leslie Wests 1969 veröffentlichtem Album Mountain, nach dem sich die daraus entstehende Formation dann auch benannte; es wurde 1972 noch einmal mit auf die Mountain-Platte The Road Goes Ever On aufgenommen. Vgl. dazu http://www.rockforever.com/singers/west/albums/mountain.html [Stand: 12. 3. 2007] sowie http://www.warr.org/ mountain.html [Stand: 12.3. 2007]. 
Dass dieser mithin aus mehr als nur der Erschießung Jay-Zs besteht, hat man offensichtlich auch bei den jährlich verliehenen MTV Video Music Awards verstanden, wo der Clip schließlich für sechs Auszeichnungen nominiert wurde und drei davon gewann. ${ }^{53}$ In einem Interview wurde Romanek darauf von dem Journalisten Reto Caduff angesprochen: „Am Schluss des Clips stirbt Jay-Z im Kugelhagel. Eine Tatsache, mit der die Zensoren bei MTV ihre liebe Mühe hatten. Nun ist das Video für sechs MTV Music Awards nominiert." Romaneks Antwort darauf ist so kurz wie lakonisch: „Lustig, nicht?" 54

\section{Anhang}

\section{Problems}

S. Carter, R. Rubin, J. Ventura, L. Weinstein [Sample of „Long Red“], W. Squier [Sample of „Big Beat"]

Produced by Rick Rubin

(C) 2003 Roc-A-Fella Records (Def Jam/Universal)

If you're havin' girl problems I feel bad for you, son

I got 99 problems but a bitch ain't one

I got the rap patrol on the gat patrol

Foes that wanna make sure my casket's closed

Rap critics that say he's „Money Cash Hoes“

I'm from the hood stupid what type of facts are those

If you grew up with holes in your zapatos

You'd celebrate the minute you was havin' dough

I'm like fuck critics you can kiss my whole asshole

If you don't like my lyrics you can press fast forward

Got beef with radio if I don't play they show

They don't play my hits well I don't give a shit so

Rap mags try and use my black ass

So advertisers can give 'em more cash for ads ... fuckers

I don't know what you take me as

or understand the intelligence that Jay- $Z$ has

I'm from rags to riches nigga I ain't dumb

I got 99 problems but a bitch ain't one

Hit me

\section{CHORUS}

99 Problems but a bitch ain't one

If you havin' girl problems I feel bad for you son

53 Der Clip war nominiert in den Kategorien „Best Direction in a Video“, „Best Cinematography in a Video“, „Best Editing in a Video“, „Best Video of the Year“, „Best Male Video“, „Best Rap Video“ und gewann am 29. August 2004 in Miami in den drei letztgenannten Sparten.

54 Caduff, „Reality-TV mit Seele“ (wie Anm. 19). 
I got 99 problems but a bitch ain't one

Hit me

The year is ' 94 and in my trunk is raw

In my rear view mirror is the mother fuckin' law

I got two choices y'all pull over the car or

Bounce on the devil put the pedal to the floor

Now I ain't tryin to see no highway chase with jake

Plus I got a few dollars I can fight the case

So I pull over to the side of the road

I heard "Son do you know what I'm stoppin' you for?"

"Cause I'm young and I'm black and my hat's real low

Do I look like a mind reader sir, I don't know

Am I under arrest or should I guess some mo"?"

"Well you was doin' fifty five in a fifty four"

License and registration and step out of the car

Are you carryin' a weapon on you I know a lot of you are"

"I ain't steppin' out of shit all my papers legit"

"Do you mind if I look 'round the car a little bit?"

"Well my glove compartment is locked so is the trunk and the back

And I know my rights so you gon' need a warrant for that"

„Aren't you sharp as a tack? are some type of lawyer or something?

Or somebody important or somethin'?"

„Nah, I ain't pass the bar but I know a little bit

Enough that you won't illegally search my shit"

"Well see how smart you are when the K-9's come"

I got 99 problems but a bitch ain't one

Hit me

\section{CHORUS}

Now once upon a time not too long ago

A nigga like myself had to strong arm a hoe

This is not a hoe in the sense of havin' a pussy

But a pussy havin' no God Damn sense, try and push me

I tried to ignore him and talk to the Lord

Pray for him, cause some fools just love to perform

You know the type - loud as a motor bike

But wouldn't bust a grape in a fruit fight

The only thing that's gonna happen is I'mma get to clappin'

He and his boys gon' be yappin' to the captain

And there I go trapped in the kit kat again

Back through the system with the riff raff again

Fiends on the floor scratchin again

Paparazzi's with they cameras snappin' them

D. A. tried to give the nigga the shaft again

Half-a-mil for bail cause I'm African

All because this fool was harassin' them 
Tryin to play the boy like he's saccharin

But ain't nothin' sweet 'bout how I hold my gun

I got 99 problems but this bitch ain't one

Hit me

\section{CHORUS}

You're crazy for this one Rick

It's your boy

Die Wiedergabe des Textes folgt der URL: http://slangcity.com/songs/ 99_problems.htm [Stand: 12. 3. 2007]. 